\title{
Sintomas de pacientes em tratamento oncológico
}

\author{
Symptoms of patients undergoing oncological treatment
}

\author{
Valdecir Brateibach ${ }^{1}$, Edvane Lopes Birelo de Domenico ${ }^{2}$, Evelise Moraes Berlezi ${ }^{3}$, Marli \\ Maria Loro ${ }^{4}$, Cleci de Lourdes Schmidt Piovesan Rosanelli ${ }^{4}$, Joseila Sonego Gomes ${ }^{5}$, \\ Adriane Cristina Bernat Kolankiewicz ${ }^{6}$
}

\section{RESUMO}

Objetivo: Associar sintomas de pacientes em tratamento oncológico com o tipo de tratamento, faixa etária e sexo.

Materiais e Métodos: Estudo observacional, transversal e analítico; realizado com 480 pacientes em tratamento atendidos em um Centro de Alta Complexidade de Tratamento para Câncer no Sul do Brasil no período de abril a dezembro de 2011.

Resultados: A média de idade dos entrevistados foi de 58,23 ( $\mathrm{DP}=13,11)$ anos. Os sintomas mais freqüentes nos pacientes em tratamento quimioterápico antineoplásico foram a fadiga e a dor com 78,5\%, o que corresponde a 238 pacientes, seguido de constipação e insônia com $51,8 \%$ e 47,5\%, respectivamente, e principalmente no sexo feminino. Na comparação das médias dos sintomas conforme o tratamento, náuseas e vômitos apresentaram associação com $p=0,013$, enquanto na comparação dos sintomas com os ciclos de vida náuseas e vômitos apresentaram $p=0,05$ e 0,24 , respectivamente.

Conclusão: $O$ estudo evidencia associação estatística dos sintomas náuseas e vômitos com o ciclo de vida e tratamento, e revela maiores queixas entre as mulheres. Estes dados podem ser utilizados na prática assistencial, auxiliando na individualização da assistência.

Palavras-chave: neoplasias; sintomas; quimioterapia; radioterapia.

\begin{abstract}
Objective: To associate symptoms of patients with cancer undergoing treatment with the type of treatment, age and gender.

Materials and Methods: Observational, cross-sectional and analytical study; conducted with 480 patients under treatment enrolled in a High Complexity Cancer Treatment Center in southern Brazil from April to December of 2011.

Results: The average age of respondents was $58.23(S D=13.11)$ years old. The most frequent symptoms in patients undergoing anticancer chemotherapy were fatigue and pain with 78.5\% (corresponding to 238 patients), followed by constipation and insomnia, with $51.8 \%$ and $47.5 \%$, respectively, and mostly in women. When comparing the symptoms average according to the treatment, nausea and vomiting presented an association with $p=0.013$, while when comparing the symptoms with the life cycles, nausea and vomiting showed an association with $p=0.05$ and 0.24 , respectively.

Conclusions: The study shows association of nausea and vomiting symptoms with the life cycle and the treatment, pointing to more complain among women. These data can be used in the health care contributing to an individualized assistance.
\end{abstract}

Keywords: neoplasms; symptoms; drug therapy; radiotherapy.

\footnotetext{
${ }^{1}$ Enfermeiro graduado pela Universidade do Noroeste do Estado do Rio Grande do Sul (UNIJUí).

${ }^{2}$ Enfermeira. Doutora em Enfermagem. Professora Adjunta do Departamento de Enfermagem Clínica e Cirúrgica da EPE (UNIFESP).

${ }^{3}$ Fisioterapeuta. Doutora em Gerontologia. Docente do Curso de Fisioterapia (UNIJUí).

${ }^{4}$ Enfermeira. Doutoranda nas Ciências pela UNIFESP. Docente do Curso de Graduação em Enfermagem (UNIJUÍ).

${ }^{5}$ Enfermeira. Mestre em Enfermagem pela Escola de Enfermagem de Ribeirão Preto (EERP/USP). Docente do Curso de Enfermagem (UNIJUÍ).

${ }^{6}$ Enfermeira. Doutoranda na Escola Paulista de Enfermagem da Universidade Federal de São Paulo (EPE-UNIFESP). Departamento de Ciências da Vida (UNIJUÍ).
} 


\section{INTRODUÇÃO}

O conceito câncer consiste num grupo heterogêneo de doenças que se caracterizam pela perda do controle da divisão celular, desencadeando o aparecimento de massas celulares anormais. Seu crescimento é desordenado, autônomo e excede o dos tecidos normais, competindo com estes quanto ao fornecimento de energia e substrato nutricional ${ }^{1}$. É considerada uma doença crônica de relevância para a saúde pública levando em consideração os vários aspectos: sua elevada incidência, prevalência, mortalidade, gastos hospitalares e, consequências para os pacientes, bem como demandas de cuidado para os profissionais de saúde ${ }^{2}$.

O câncer é a segunda principal causa de morte nos países desenvolvidos e a terceira nos países em desenvolvimento. Estima-se que ele é responsável por $12,6 \%$ do total de mortes em todo o mundo. Esta estimativa é superior ao número de mortes causadas pela Síndrome da Imunodeficiência Adquirida (HIV/SIDA), tuberculose e malária juntos no mundo ${ }^{3}$. 0 Instituto Nacional do Câncer - INCA, órgão do Ministério da Saúde que traça diretrizes para o controle e tratamento do câncer estimou que para os próximos anos pudessem ocorrer 489.270 mil casos novos de câncer no país, sendo que cerca de 49.240 mil destes casos seriam de câncer de mama e 60.180 mil de câncer de próstata ${ }^{4}$. O seu número de casos novos estimado para 2012-2013 é de 518.510 no Brasil, incluindo os casos de câncer de pele não melanoma, sendo este o tipo mais incidente em ambos os sexos com 134 mil casos novos, seguido do câncer de próstata (60 mil), mama feminina (53 mil), colón e reto (30 mil), pulmão (27 mil), estômago (20 mil) e colo de útero $(18 \mathrm{mil})^{5}$.

O diagnóstico e tratamento do câncer requerem atenção de uma equipe multiprofissional, altamente especializada, na medida em que utiliza geralmente, tecnologia sofisticada e dispendiosa. Por ser doença de natureza crônica possui alto custo econômico, pois pode provocar incapacidades, exige internação hospitalar e acompanhamento ambulatorial frequentes ${ }^{1}$. No tratamento do câncer faz-se necessário a continua avaliação da evolução do mesmo, porém essas investigações precisam ir além das características físicas do indivíduo e aspectos do tumor. O tratamento é complexo e inclui várias modalidades de tratamento entre eles a cirurgia, a hormonioterapia, a radioterapia $(\mathrm{RxT})$, a quimioterapia (QT), terapia alvo específica ou pela combinação de duas ou mais terapias ${ }^{6}$.

A QT é o tratamento mais comum e constitui-se de um conjunto de drogas que atua em várias fases do metabolismo celular, atingindo além de células malignas, as células sadias do organismo, sendo responsável por diversas reações/sintomas como: anemia, fadiga, leucopenia, apatia, perda de apetite, alopecia, diarréia, perda de peso, mucosites, hematomas, náuseas e vômitos. Todos estes fatores são causadores de desconforto, estresse e sofrimento, além de possíveis internações prolongadas ${ }^{7}$.

A RxT é a terapia de escolha para os tumores malignos sensíveis à irradiação ionizante, ou seja, aos danos moleculares capazes de inibir a clonogenicidade das células malignas ${ }^{2}$. A RxT é a modalidade indicada para tumores localizados que não podem ser ressecados totalmente ou em tumores que costumam recidivar localmente após o tratamento cirúrgico. Dentre os sintomas causados pela a RxT, a mucosite oral por radiação é uma manifestação bastante freqüente durante $\mathrm{e}$, até alguns dias após $\mathrm{o}$ tratamento. $\mathrm{O}$ paciente refere dor intensa, dificuldade para se alimentar e realizar higiene oral e falar, culminando, muitas vezes na interrupção do tratamento até a recuperação do processo inflamatório ${ }^{8}$. Na RxT, um dos eventos que possui mais relevância decorrente da radiação ionizantes são as lesões de pele denominadas de radiodermite, as quais podem ser agudas ou tardias e comprometem a qualidade de vida do indivíduo, devido à alteração da imagem corporal, auto-imagem, auto-estima, levando ao isolamento social ${ }^{9}$.

A QT tem grande importância no tratamento pósoperatório e combinada à radioterapia auxilia no controle e no aparecimento de metástases no $\operatorname{organismo}^{10}$. Entre os efeitos colaterais os mais freqüentemente observados são: diminuição das células sanguíneas, mucosite e alopecia $^{10}$. Em pacientes oncológicos, independentemente do tratamento realizado, a fadiga é um sintoma comum que pode ser 
definido como uma persistente e subjetiva sensação de cansaço $^{9}$. Possui como característica a falta de energia, exaustão, perda de interesse por atividades anteriormente prazerosas, fraqueza, dispnéia, dor, alterações de paladar, prurido, lentidão, irritabilidade e perda da concentração ${ }^{11}$.

Nessa perspectiva, este estudo tem por objetivo geral associar os sintomas de pacientes em tratamento oncológico com o tipo de tratamento, faixa etária e gênero; e, como objetivos específicos: identificar o perfil sócio-demográfico dos pacientes oncológicos em tratamento radioterápico, quimioterápico ou conjugado.

\section{MATERIAIS E MÉTODOS}

Este estudo é parte da pesquisa institucional "Qualidade de vida de pacientes oncológicos assistidos no ambulatório do Centro de Alta Complexidade para o Tratamento do Câncer (CACON) desenvolvido pela Universidade Regional do Noroeste do Rio Grande do Sul (UNIJUÍ) e trata-se de um estudo observacional, transversal e analítico.

Fizeram parte da amostra do estudo pacientes oncológicos, atendidos no ambulatório do CACON do Hospital de Caridade de ljuí ( $\mathrm{HCl}$ ), hospital de porte IV, localizado na região noroeste do Estado do Rio Grande do Sul/Brasil. Para a composição da amostra foram considerados os seguintes critérios de inclusão: paciente em tratamento por QT intravenosa e RxT ou conjugado; no caso dos pacientes em tratamento quimioterápico considerou-se para a entrada ter realizado no mínimo três ciclos de QT de qualquer protocolo; no caso dos pacientes em tratamento radioterápico, considerou-se a partir da primeira sessão. Foram excluídos os participantes de protocolos de pesquisa clínica; pacientes com alterações cognitivas sem condições de responder aos instrumentos, atestadas no prontuário.

O tamanho da amostra foi calculado a partir de dados prévios do número de pacientes atendidos no ambulatório no mês que antecedeu a coleta de dados, março de 2011, que foi de 1756. Considerando a confiabilidade de $95 \%$, erro de $5 \%$, incluindo $5 \%$ para possíveis perdas, obteve-se uma amostra de 480 pacientes, com uma representatividade de $25 \%$ da população.

A coleta de dados foi realizada no período de abril de 2011 a dezembro de 2011. As variáveis foram obtidas por meio da análise documental do prontuário do paciente, entrevista semi-estruturada e aplicação de protocolo. Do prontuário foram obtidas as informações sobre o tipo de câncer e tratamento; a entrevista abordou as questões sociodemográficas. Os sintomas foram identificados por meio da utilização do instrumento de Qualidade de Vida proposto pela European Organization for Research and treatment of Cancer - o Quality of Life Core-30-Questionnaire (EORTC QLQ-C30), validado para o português ${ }^{12}$, do qual se usou exclusivamente a identificação de sintomas. A aplicação do instrumento ocorreu no espaço físico do CACON, na ocasião em que os pacientes acessavam o serviço para a terapia e priorizou-se o momento anterior a infusão entre os ciclos.

Para a análise estatística foi utilizado o programa Statistical Package for the Social Sciences (SPSS-PC). Para a análise das variáveis qualitativas utilizou-se medidas de frequência. Para as quantitativas medidas de tendência central, medidas de dispersão e teste de comparação de médias (teste para amostras nãoparamétricas e independentes - teste de MannWhitney). Nos testes analíticos considerou-se uma confiabilidade de $95 \%$ e estatisticamente significativo valor de $p \leq 0,05$.

A pesquisa foi aprovada pelo Comitê de Ética em Pesquisa da UNIJUÍ, sob o parecer consubstanciado $\mathrm{n}^{\circ}$. 275/2010.

\section{RESULTADOS}

Dos 480 pacientes que fizeram parte do estudo a média de idade foi de 58,23 $\pm 13,11$ anos, com idade mínina de 21 e a máxima de 88 . O intervalo de confiança mostrou que $95 \%$ da população do estudo, encontram-se entre 57,05 a 59,40 anos de idade. Com relação aos dados sócio-demográficos destaca-se que a maioria era do sexo feminino (53,73\%), casados $(66,9 \%)$, baixa escolaridade $(68,4 \%)$ e renda $(63,1 \%)$ e de cor branca $(88,3 \%)$ conforme dados da tabela 1 . 
TABELA 1 - Descrição da amostra $(n=480)$ segundo condições sócio-demográficas investigadas.

\begin{tabular}{|c|c|c|}
\hline Variável & $\mathbf{N}$ & $\%$ \\
\hline \multicolumn{3}{|l|}{ Idade } \\
\hline Adulto (21 a 59 anos) & 248 & 51,7 \\
\hline Idoso ( $\geq 60$ anos) & 232 & 48,3 \\
\hline \multicolumn{3}{|l|}{ Gênero } \\
\hline Masculino & 222 & 46,25 \\
\hline Feminino & 258 & 53,75 \\
\hline \multicolumn{3}{|l|}{ Escolaridade (níveis) } \\
\hline Sem Instrução & 3 & 0,6 \\
\hline Ensino Fundamental Incompleto & 328 & 68,3 \\
\hline Ensino Fundamental Completo & 39 & 8,1 \\
\hline Ensino Médio Incompleto & 18 & 3,7 \\
\hline Ensino Médio Completo & 61 & 12,7 \\
\hline Ensino Superior & 31 & 6,4 \\
\hline \multicolumn{3}{|l|}{ Estado Civil } \\
\hline Casado & 321 & 66,9 \\
\hline Solteiro & 41 & 8,5 \\
\hline Viúvo & 57 & 11,9 \\
\hline Concubinado & 18 & 3,8 \\
\hline Separado & 42 & 8,8 \\
\hline \multicolumn{3}{|l|}{ Cor } \\
\hline Branco & 424 & 88,3 \\
\hline Negro & 23 & 4,8 \\
\hline Mulato & 33 & 6,9 \\
\hline
\end{tabular}

Renda*

$\begin{array}{lcc}\text { Abaixo de } 1 \text { Salário Mínimo } & 22 & 4,6 \\ 1 \text { a } 2 \text { Salários Mínimos } & 303 & 63,1 \\ 3 \text { a } 5 \text { Salários Mínimos } & 104 & 21,7 \\ 5 \text { a } 8 \text { Salários Mínimos } & 21 & 4,4 \\ \text { Acima de } 8 \text { Salários Mínimos } & 13 & 2,7 \\ \text { Total } & \mathbf{4 8 0} & \mathbf{1 0 0}\end{array}$

Renda foi considerada a válida em março de $2011 \mathrm{R} \$: 545,00$ (Quinhentos e quarenta e cinco reais).

Os tipos de cânceres dos pacientes entrevistados foram: câncer de mama (23,3\%), gastrointestinal $(23,3 \%)$, de aparelho reprodutor feminino (14,8\%), de pele, músculos e ossos $(10,8)$, reprodutor masculino $(8,8 \%)$, do aparelho respiratório (7,3\%), linfomas, mielomas e leucemia $(6,7 \%)$ e outros $(0,6 \%)$.

Os estádios clínicos (EC) ao diagnóstico destes pacientes eram: $6 \%$ foram diagnosticados em EC I, $24 \%$ em EC II, 30\% em EC III, 26\% em EC IV e 14\% não apresentavam esta informação no prontuário.

Observou-se que os sintomas mais frequentes, nos pacientes em tratamento QT antineoplásico, foram a fadiga e a dor com $78,5 \%$, o que corresponde a 238 pacientes, seguido de constipação e insônia com 51,8\% e $47,5 \%$ respectivamente (tabela 2 ).

Dos pacientes em tratamento RxT as prevalências de sintomas foram menores do que as do tratamento QT, porém maiores do que no tratamento conjugado. Assemelham-se os sintomas mais freqüentes do tratamento QT com os sintomas mais freqüentes fadiga, dor e insônia com 72,4\%, $71,6 \%$ e $47,4 \%$ respectivamente. E no tratamento QT e RxT verificou-se a fadiga e a dor, seguidas da falta de apetite com $82 \%$, $82 \%$ e $52,5 \%$ respectivamente (tabela 2 ).

Evidencia-se que os sintomas mais presentes manifestaram-se na população adulta, exceto a dispnéia. Os sintomas mais freqüentes na população adulta foram: fadiga, a dor e a constipação com $77,4 \%$, $77 \%$ e $48,4 \%$. E na população idosa permanecem os mesmos sintomas, porém com frequências menores, a fadiga e a dor com os mesmos escores $77,6 \%$ e constipação com 46,6\% (tabela 3).

Quando analisados os sintomas a partir do sexo, as frequências maiores de todos os sintomas foram no sexo feminino, sendo que a fadiga, dor e constipação ocuparam os maiores índices (tabela 4).

Ao comparar as médias dos sintomas conforme os ciclos de vida foram estatisticamente associados os sintomas de náuseas/vômitos e insônia (tabela 5). E ao comparar as médias dos sintomas conforme o sexo ficou estatisticamente associado os sintomas de náuseas/vômitos (tabela 6).

\section{DISCUSSÃo}

O presente estudo estimou a prevalência de sintomas apresentados pelos pacientes em tratamento oncológico, bem como as correlações destes com o tipo de tratamento, idade e gênero. As maiores freqüências da doença foram de câncer de mama, gastroinstestinal e de aparelho reprodutor feminino. O Brasil está em 
TABELA 2 - Prevalência de sintomas conforme o tipo de tratamento.

\begin{tabular}{lccc}
\hline Sintoma - n (\%) & QT & RxT & QT e RxT \\
\hline Fadiga & $238(78,5)$ & $84(72,4)$ & $50(82)$ \\
Naúseas (Vômito) & $131(43,2)$ & $34(29,3)$ & $32(52,5)$ \\
Dor & $238(78,5)$ & $83(71,6)$ & $50(82)$ \\
Dispnéia & $73(24,2)$ & $21(18,1)$ & $10(16,4)$ \\
Insônia & $144(47,5)$ & $55(47,4)$ & $26(42,6)$ \\
Perda de Apetite & $115(38)$ & $47(40,5)$ & $32(52,5)$ \\
Constipação & $157(51,8)$ & $45(38,8)$ & $26(42,6)$ \\
Diarréia & $50(16,5)$ & $23(19,8)$ & $14(23,0)$ \\
\hline
\end{tabular}

TABELA 3 - Prevalência de sintomas conforme os ciclos de vida.

\begin{tabular}{lcc}
\hline Sintoma - n (\%) & Adulto & Idoso \\
\hline Fadiga & $192(77,4)$ & $180(77,6)$ \\
Náuseas (Vômito) & $117(47,2)$ & $80(34,5)$ \\
Dor & $191(77)$ & $180(77,6)$ \\
Dispnéia & $50(20,2)$ & $54(23,4)$ \\
Insônia & $124(50)$ & $101(43,5)$ \\
Perda de Apetite & $101(40,7)$ & $93(40,1)$ \\
Constipação & $120(48,4)$ & $108(46,6)$ \\
Diarréia & $46(18,5)$ & $41(17,7)$ \\
\hline
\end{tabular}

TABELA 4 - Prevalência de sintomas do sexo masculino e feminino.

\begin{tabular}{lcc}
\hline Sintoma - n (\%) & Masculino & Feminino \\
\hline Fadiga & $168(75,7)$ & $204(79,1)$ \\
Náuseas (Vômito) & $80(36,0)$ & $117(45,3)$ \\
Dor & $167(75,2)$ & $204(79,1)$ \\
Dispnéia & $49(22,2)$ & $55(21,3)$ \\
Insônia & $108(48,6)$ & $117(45,3)$ \\
Perda de Apetite & $84(37,8)$ & $110(42,6)$ \\
Constipação & $101(45,5)$ & $127(49,2)$ \\
Diarréia & $41(18,5)$ & $46(17,8)$ \\
\hline
\end{tabular}

uma fase de transição demográfica, na qual há quedas nas taxas de natalidade e mortalidade, coexistindo, nesse cenário, com a ocorrência de doenças agudas e crônicas, de cânceres em crianças, adultos, jovens e idosos e de cânceres de países ricos e pobres ${ }^{13}$.

No que tange ao desenvolvimento socioeconômico, o câncer de estômago e de colo uterino são indicadores de saúde de sociedades menos desenvolvidas, enquanto o câncer de mama e de pulmão, de sociedades mais desenvolvidas ${ }^{13}$.

Prevaleceram no estudo, adultos do sexo feminino, casadas, brancas com baixa escolaridade e renda. A faixa etária é o fator de risco importante, dependendo do tipo de câncer, podendo representar 0 efeito acumulativo da exposição, ao longo da vida, aos agentes carcinogênicos ${ }^{14}$.

Os dados sócio-demográficos desta pesquisa corroboram com os dados da investigação de outro estudo, em que $90 \%$ dos participantes apresentavam os estudos primários, completo ou incompleto como escolaridade e 6,6\% apresentavam curso universitário ${ }^{15}$, assim como em outro estudo a menor parte $26,68 \%$ dos pacientes investigados tinham nível superior de escolaridade $^{16}$. Pesquisadores apontam em estudo realizado que $89,5 \%$ dos sujeitos da pesquisa eram de nível socioeconômico médio ou baixo, estando de acordo com os dados da nossa pesquisa ${ }^{17}$. Estudo sobre a prevalência das doenças crônicas na população brasileira constatou que a desigualdade de condições de vida da população adulta brasileira, avaliada pelos anos de escolaridade, associa-se a diferentes prevalências de condições crônicas, sendo as mais elevadas constatadas, em geral, nos segmentos socialmente mais desfavorecidos ${ }^{18}$. Dados que vem ao encontro do nosso estudo.

O tratamento do câncer dependerá do estadiamento, do tamanho do tumor e de fatores pessoais. A terapêutica poderá consistir em extirpação do tumor, retirada de linfonodos e terapia coadjuvante como QT, Rxt e hormonioterapia, de acordo com a indicação, todos com a finalidade de combater o carcinoma e evitar a disseminação da doença, destruindo as células cancerosas $^{19}$.

Sobre os sintomas apresentados pelos pacientes no decorrer do curso da doença, a dor e a fadiga foram os sintomas mais presentes na vigência do tratamento QT, na presente investigação. Dos pacientes em tratamento RxT as prevalências de sintomas foram menores do que as do tratamento $\mathrm{QT}$, porém maiores do que no tratamento conjugado. Esse fato pode ser em 
TABELA 5 - Médias e desvio padrão conforme o ciclo de vida.

\begin{tabular}{|c|c|c|c|c|}
\hline Sintomas & Ciclos de Vida & Média & Desvio Padrão & Valor de $p$ \\
\hline \multirow[t]{2}{*}{ Fadiga } & Adulto & 29,65 & 26,56 & 0,333 \\
\hline & Idoso & 27,92 & 27,03 & \\
\hline \multirow[t]{2}{*}{ Naúseas/Vômito } & Adulto & 17,63 & 24,96 & 0,005 \\
\hline & Idoso & 12,38 & 22,00 & \\
\hline \multirow[t]{2}{*}{ Dor } & Adulto & 30,13 & 32,50 & 0,375 \\
\hline & Idoso & 27,72 & 31,82 & \\
\hline \multirow[t]{2}{*}{ Insônia } & Adulto & 32,53 & 37,74 & 0,024 \\
\hline & Idoso & 23,96 & 32,32 & \\
\hline \multirow[t]{2}{*}{ Dispnéia } & Adulto & 10,87 & 10,87 & 0,500 \\
\hline & Idoso & 11,17 & 11,17 & \\
\hline \multirow[t]{2}{*}{ Falta de apetite } & Adulto & 22,81 & 32,56 & 0,942 \\
\hline & Idoso & 23,81 & 34,36 & \\
\hline \multirow[t]{2}{*}{ Constipação } & Adulto & 27,66 & 33,90 & 0,692 \\
\hline & Idoso & 26,42 & 33,17 & \\
\hline \multirow[t]{2}{*}{ Diarréia } & Adulto & 9,66 & 22,95 & 0,892 \\
\hline & Idoso & 10,61 & 26,34 & \\
\hline
\end{tabular}

decorrência de uma intencionalidade paliativa de tratamento, no qual tanto o cálculo da dose dos quimioterápicos como da radiação são reduzidos para que haja controle do crescimento do tumor e minimização dos sinais e sintomas e não a intencionalidade de cura da doença neoplásica, considerando que a maior parte da amostra deste estudo estava entre os estádios clínicos III e IV.

A dor é um sintoma mais freqüente nas neoplasias malignas e, muitas vezes, o sintoma mais temido, mas esta dor pode ser reduzida significativamente se houver uma avaliação e tratamento adequados. Os sintomas álgicos somam-se às incapacidades relacionadas à neoplasia e seu tratamento e pode causar insônia, anorexia, confinamento no leito, isolamento social o que acarreta grande estresse e sofrimento ao doente e aos que o rodeiam ${ }^{20}$.

A fadiga é um dos sintomas mais frequentes e debilitantes, que pode ocorrer durante o tratamento, após o seu término ou em estágios avançados da doença e, não há melhora após o repouso, tornando-se crônico e causando limitação nas atividades diárias ${ }^{20}$. A etiologia da fadiga após o tratamento de câncer ainda permanece incerta. Estudos apontam que um declínio da hemoglobina durante as repetidas administrações de quimioterapia é acompanhado de aumento na fadiga e declínio na $\operatorname{cognição}^{21}$. A fadiga é relatada simultaneamente com outros sintomas como dor, falta de sono, entre outros. Pode ser considerado o pior dos sintomas dos portadores de câncer e por sua alta prevalência (50 a 90\%) e pelas consequências que ocasiona na vida da população, deveria ser mais valorizada pelos profissionais ${ }^{20}$. De acordo com estudos os mecanismos que produzem a fadiga são desconhecidos, estudos tem relacionado à fadiga com as consequências da doença que dependem do tratamento e do momento do tratamento ${ }^{22}$. Alguns mecanismos somáticos têm sido relacionados com a fadiga como a desnutrição provocada pela anorexia, mudanças de metabolismo, vômitos, diarréia; a anemia é um outro fator que pode levar ao cansaço durante o dia, que pode interferir na qualidade de vida dos pacientes $^{22}$.

As manifestações podem incluir diminuição do autocuidado, capacidade física, memória e concentração, falta de interesse e motivação nas atividades, fraqueza, irritabilidade, frustração, tristeza e angústia espiritual ${ }^{20}$. Estas caracterizam a 
TABELA 6 - Comparação de médias das amostras não paramétricas e independentes conforme o sexo.

\begin{tabular}{|c|c|c|c|c|}
\hline Sintomas & Sexo & Média & Desvio Padrão & Valor de $p$ \\
\hline \multirow[t]{2}{*}{ Fadiga } & Masculino & 26,06 & 25,08 & 0,056 \\
\hline & Feminino & 31,18 & 27,99 & \\
\hline \multirow[t]{2}{*}{ Naúseas/Vômito } & Masculino & 11,88 & 20,45 & 0,013 \\
\hline & Feminino & 17,86 & 25,89 & \\
\hline \multirow[t]{2}{*}{ Dor } & Masculino & 26,37 & 31,46 & 0,082 \\
\hline & Feminino & 31,20 & 32,66 & \\
\hline \multirow[t]{2}{*}{ Dispnéia } & Masculino & 11,55 & 24,41 & 0,758 \\
\hline & Feminino & 10,56 & 23,50 & \\
\hline \multirow[t]{2}{*}{ Insônia } & Masculino & 28,95 & 35,30 & 0,622 \\
\hline & Feminino & 27,90 & 35,65 & \\
\hline \multirow[t]{2}{*}{ Falta de apetite } & Masculino & 20,68 & 31,26 & 0,173 \\
\hline & Feminino & 25,55 & 35,07 & \\
\hline \multirow[t]{2}{*}{ Constipação } & Masculino & 24,44 & 31,54 & 0,185 \\
\hline & Feminino & 29,31 & 35,05 & \\
\hline \multirow[t]{2}{*}{ Diarréia } & Masculino & 10,79 & 26,01 & 0,799 \\
\hline & Feminino & 9,54 & 23,41 & \\
\hline
\end{tabular}

multidimensionalidade do sintoma, ou seja, o impacto em diversos âmbitos da vida do paciente. Estudo aponta que há relativo prejuízo nas funções física, emocional, cognitiva e social ante o aumento dos sintomas fadiga, náuseas e vômitos, dor, e insônia ${ }^{23}$. Esses sintomas são os mais prevalentes e podem interferir negativamente na qualidade de vida $(\mathrm{QV})$ do paciente em tratamento, uma vez que está diretamente relacionado ao bem estar do mesmo.

As pacientes do sexo feminino são as mais acometidas pelos sintomas, principalmente pela náusea e vômito. As alterações gastrintestinais provocadas pela QT são constantes e, as mulheres demonstram maior suscetibilidade a estes sintomas sendo, muitas vezes, mas difícil o distanciamento destas das funções relacionadas ao preparo dos alimentos que, sabidamente, agravam a sintomatologia. Culturalmente, as mulheres também apresentam menor pudor para expressar sinais e sintomas que, para os homens, pode ser intrepretado como fraqueza ${ }^{24}$.

Nesse contexto complexo de manifestações diversificadas e dependentes de variáveis, o papel da equipe de saúde, em especial da enfermagem, por ser o profissional que mais permanece junto ao paciente, é complexo e necessita ser pautado em evidências científicas para que as demandas dos pacientes possam ser satisfeitas, mesmo em estruturas e processos assistenciais que dificultem esta individualização em razão da insuficiência de recursos, tanto humanos como materiais. A Enfermagem exerce papel importante no controle dos efeitos adversos e nas consequências do tratamento sobre o desempenho físico, psicológico e social do paciente ${ }^{15}$.

Este estudo tem uma limitação importante, tendo em vista que a população estudada não ter sido homogênea quanto uso de mesmos protocolos de QT e também por terem sido entrevistados somente pacientes em RxT e QT, excluindo os demais tipos de tratamento.

Foram entrevistados 480 pacientes, sendo a maioria do sexo feminino, casados, de baixa escolaridade e renda e de cor branca. Os estádios clínicos, na maioria dos casos, eram avançados ao diagnóstico. Os sintomas mais frequentes, nos pacientes em tratamento QT antineoplásico foram a fadiga e a dor, seguido de constipação e insônia. Dos pacientes em tratamento RxT as prevalências de sintomas foram menores do que 
as do tratamento QT, porém maiores do que no tratamento conjugado. $\mathrm{E}$ no tratamento QT e RxT seguem os mais frequentes fadiga e dor seguido de falta de apetite. Evidencia-se que os sintomas mais presentes manifestaram-se na população adulta $e$ feminina. Os resultados revelaram associação estatística significativa entre os ciclos de vida e sexo com o sintoma de náuseas e vômitos. Os resultados suscitam a análise da importância de ações em saúde, prevenção e manejo adequadas aos sintomas, de forma sistemática, considerando-se as modalidades de tratamento, faixa etária e sexo.

\section{REFERÊNCIAS}

1. Brasil. Ministério da Saúde. Instituto Nacional de Câncer (INCA). Ações de enfermagem para o controle do câncer: uma proposta de integração ensino-serviço. $3^{\text {a }}$. ed. Rio de Janeiro: MS/INCA; 2008.

2. Herr GH, Kolankiewicz ACB, Berlezi EM, Gomes JS, Magnago TSBS, Rosanelli CP, Loro MM. Avaliação de conhecimentos acerca da doença oncológica e práticas de cuidado com a saúde. Rev Bras de Cancerol. 2013; 59(1):33-44.

3. Simino GPR, Santos CB, Mishima SM. Follow-up of Cancer Patients by Family Health Workers. Rev Latino-Am Enfermagem 2010;18(5):856-63.

4. Brasil. Ministério da Saúde. Instituto Nacional Do Câncer. Orientações aos pacientes sobre radioterapia. 2010. [capturado 2012 fev.]. Disponível em: www.inca.gov.br/ inca/Arquivos/manuais/radioterapia.pdf

5. Brasil. Ministério da Saúde. Instituto Nacional do Câncer. Estimativa 2012 - Incidência de Câncer no Brasil. [capturado 2011 dez 20]. Acesso em: http://www1.inca.gov.br/rbc/n_57/v04/pdf/13_resenha_esti mativa2012_incidencia_de_cancer_no_brasil.pdf

6. Brasil. Ministério da Saúde. Instituto Nacional do Câncer. Tratamento para o câncer: radioterapia e quimioterapia. 2008. [capturado 2012 mar.]. Disponível em: www.inca.gov.br/enfermagem/docs.

7. Pedro ENR, Funghetto SS, Concepções de cuidado para os cuidadores: um estudo com a criança hospitalizada com câncer. Rev Gaúcha Enferm 2005;26(2):210-9.

8. Albuquerque I, Camargo T. Prevenção e tratamento da mucosite oral induzida por radioterapia: revisão de literatura. Rev Bras de Cancerol 2007;53(2):195-209.

9. Blecha F, Santos GMT. Tratamento de radiodermite no cliente oncológico: subsídios para intervenções de enfermagem. Rev Bras Cancerol. 2006;52(2):151-63.

10. Basso J. Análise postural através da biofotogrametria em mulheres mastectomizadas (trabalho de conclusão de curso). ljuí (RS): Universidade Regional do Noroeste do Estado do Rio Grande do Sul; 2007.

11. Brasil. Ministério da Saúde. Instituto Nacional do Câncer. Manual para o tratamento do câncer. [capturado 2012 fev.]. Disponível em: www.fef.br/biblioteca/arquivos/ data/acoescap6.pdf.

12. EORTEC, EORTEC QLQ C-30. 2011. [capturado 2013 abr. 4]. Disponível em: http://www.eortc.be/qol/files/ C30/QLQ-C30\%20PortugueseBrazilian.pdf

13. Menezes CNB, Passareli PM, Drude FS, Santos MA, Valle ERM. Câncer infantil: organização familiar e doença. Rev Mal Estar e Subjetividade 2007;7(1):191-210.

14. Juberg $C$, Gouveia ME, Belisário $C$. Na mira do câncer: o papel da mídia brasileira. Rev Bras de Cancerol. 2006; 52(1):139-46.

15. Sawada NO, Nicolussill AC, Okinolll L, Cardozo FMC, Zago MMF. Avaliação da qualidade de vida de pacientes com câncer submetidos à quimioterapia. Rev Esc de Enferm USP. 2009;43(3):581-7.

16. Zapata CS, Romero HG. Calidad de vida y factores asociados en mujeres con cáncer de mama, inscritas en Antioquia, Colombia. Rev Panam Saúde Pública. 2010;28 (1):9-18.

17. Machado S, Sawada NO. Avaliação da qualidade de vida de pacientes oncológicos em tratamento quimioterápico adjuvante. Rev Esc de Enferm USP 2009;43(3):423-9.

18. Barros MBA, Francisco PMSB, Zanchetta LM, Cesar CLG. Tendências das desigualdades sociais e demográficas na prevalência de doenças crônicas no Brasil, PNAD: 20032008. Ciênc Saúde Colet. 2011;16(9):3755-68.

19. Brasil. Ministério da Saúde. Instituto Nacional do Câncer. Programa Nacional de Controle de Câncer de Colo do Útero. [capturado 2013 mar. 29]. Disponível em: http://www2.inca.gov.br/wps/wcm/connect/acoes program a_nacional_controle_cancer_colo_utero/tratamento.

20. Associação Brasileira de Cuidados Paliativos. Consenso Brasileiro de Fadiga. Rev Bras Cuidados Paliativos. 2010; 3(2,Supl 1):1-32.

21. Ishikawa NM, Derchain SFM, Thuler LCS. Fadiga em pacientes com câncer de mama em tratamento adjuvante. Rev Bras Cancerol. 2005;51(4):313-8.

22. Coelho F, Sawada NO. A fadiga nos pacientes com câncer de laringe. Rev Latino Am-Enferm 2000;7(5):103-7.

23. Chaves PL. Avaliação da qualidade de vida do paciente com câncer colorretal em quimioterapia ambulatorial [dissertação]. Porto Alegre (RS): Escola de Enfermagem, Programa de Pós-Graduação, UFRGS; 2010.

24. Perez GH, Nicolau JC, Bellkiss WR, Laranjeira R. Depressão e síndromes isquêmicas miocárdicas instáveis: diferenças entre homens e mulheres. Arq Bras Cardiol 2005;85(5):319-26.

\section{Endereço para correspondência:}

Adriane Cristina Bernat Kolankiewicz

Rua Tiradentes $\mathrm{n}^{\circ} 231$

ljuí/RS - CEP 98700-000

Telefone: +555581116030

E-mail: adriane.bernat@unijui.edu.br 